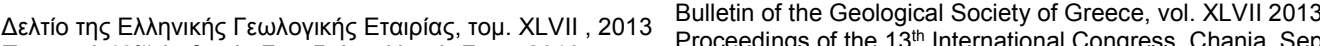

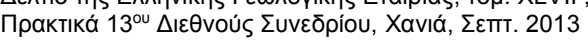
2013

\title{
COMPARATIVE EVALUATION OF ROCK-EVAL AND ELEMENTAL ANALYSIS TO DETERMINE ORGANIC CARBON CONTENT IN SEDIMENT SAMPLES
}

\author{
Pyliotis I. ${ }^{1}$, Hamilaki E. ${ }^{2}$, Pasadakis N. ${ }^{2}$ and Manoutsoglou E. ${ }^{1}$ \\ ${ }^{I}$ Technical University of Crete, Department of Mineral Resources Engineering, Research Unit of \\ Geology,Chania,73100,Greece,ipyliotis@isc.tuc.gr,emanout@mred.tuc.gr \\ ${ }^{2}$ Technical University of Crete, Department of Mineral Resources Engineering, Research Unit of \\ Hydrocarbon Chemistry and Technology, Chania, 73100,Greece, ehamilak@mred.tuc.gr, \\ pasadaki@mred.tuc.gr
}

\begin{abstract}
Rock-Eval and Elemental Analysis techniques are widely used in Organic Geochemistry for the determination of the organic content in sediment samples. Both techniques determine the carbon content using thermal treatment and pyrolysis and/or oxidation reactions. Due to the complex nature of the geochemical samples (different mineralogical composition, low organic carbon content, e.t.c) and the differences of their operational principles, disagreements are commonly observed between the analytical results of these techniques. In this work we studied in a systematic manner the performance of both techniques on a common sample set, consisting of immature, poor in organic carbon, sediments. It was demonstrated that both techniques applied on original and acid-treated samples, provide consistent analytical results for carbon content; that has also been showed by its mass-balance calculations showed.

Key words: Carbonates, acid-treatment, mass-balance.
\end{abstract}

\section{Пєрí $\eta \psi \eta$}

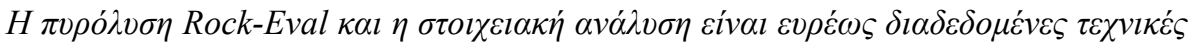

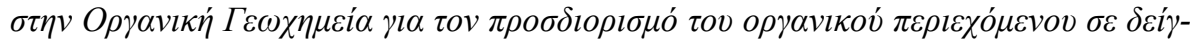

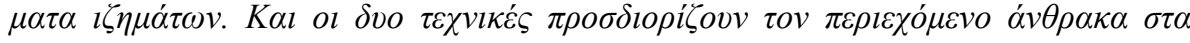

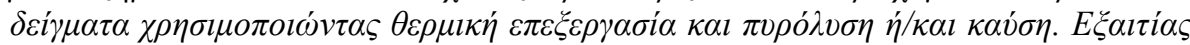

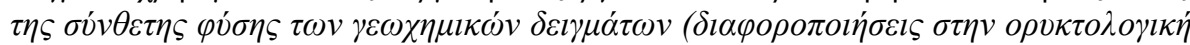

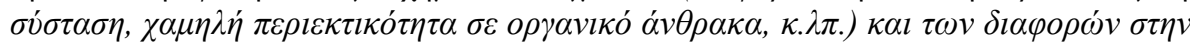

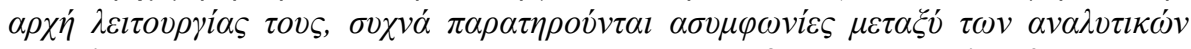

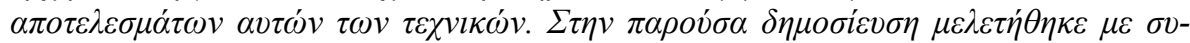

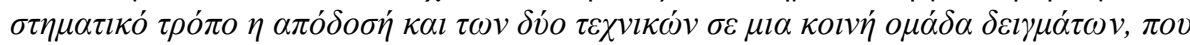

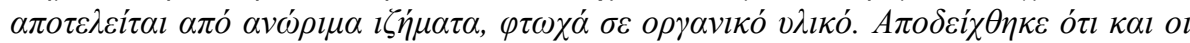

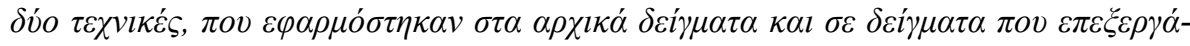

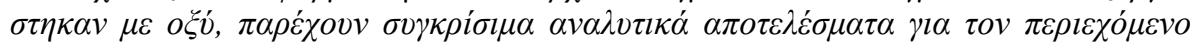

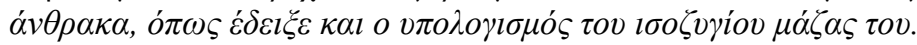

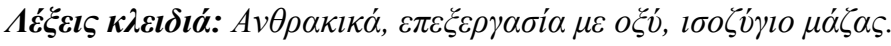

XLVII. No $2-862$ 


\section{Introduction}

Rock-Eval (RE) pyrolysis (Espitalie et al 1977) is probably the most widespread analytical technique used for the estimation of the organic matter content in sediments and provides the basic quantitative data for further geochemical analyses. RE enables the rapid screening of sediment samples, without the tedious preliminary kerogen isolation, and the reliable estimation of the organic content in terms of already existing hydrocarbons (S1), pyrolyzable organics (S2), oxygen content (S3), remaining char after pyrolysis (S4) as well as of the Total Organic Carbon (TOC) present. The values of S1, S2, S3 and S4 peaks reflect the organic matter type and content as well as the time and temperature the rock sample has undergone in the subsurface. Although the development of Rock-Eval 6 systems, with their more sophisticated detection system and higher pyrolysis/oxidation temperatures, provides more accurate estimation of the quality and quantity of organic matter (Behar et al, 2001, Lafargue et al, 1998), the well-known Rock-Eval II systems with TOC module serve, till now, in numerous petroleum laboratories worldwide.

Carbon determination in sediments based on its oxidation in an oxygen atmosphere at elevated temperatures has been also widely used in organic geochemical laboratories. Today this analysis is easy to carry out using Elemental Analysers (EA), that enables the simultaneous determination of multiple elements such as hydrogen, nitrogen, sulphur, oxygen e.t.c. Due to the high oxidation temperatures used in EA the carbon determined from sediment samples comes from both organic matter as well as from carbonates that thermally dissociate during the analysis.

It has been recognized from the early days of RE use that the obtained analytical data, especially when used to assess the type of the organic matter, may be influenced by the inorganic matrix of the sample, thus leading to questionable conclusions (Katz 1983). For instance, the presence of carbonate minerals in the samples would release $\mathrm{CO}_{2}$ during $\mathrm{RE}$ analysis, resulting in an increase of S3 values. Therefore, acidification of solid samples to remove inorganic from organic carbon is a widely encountered procedure in organic geochemistry, usually applied to sediments in order to improve the accuracy, especially of the S3 peak determination.

The aim of the present work was firstly to evaluate whether or not RE and EA techniques display comparable results and secondly to examine the effect of the presence of carbonate minerals in the determination of organic carbon content. In order to achieve these objectives, both techniques were applied on a sample set of immature, poor in organic carbon, sediments as well as on the acidtreated decarbonated counterparts.

\section{Samples and Methods}

A set consisting of twenty sediment samples, obtained from cuttings of a well penetrating neogene formations were used. The positions of the samples in the penetrated lithostratigraphic column are shown in Figure 1. The samples, after washing, to remove possible organic contaminants from drilling mud, were dried at $105^{\circ} \mathrm{C}$ overnight, crashed and sieved through a 60 mesh sieve. Their analysis was carried out according to the following protocol:

1. Aliquots of sediments $(\sim 100 \mathrm{mg})$ were analyzed in an RE II-TOC (Delsi Inc.) system connected to an $\mathrm{A} / \mathrm{D}$ acquisition system, SRI-302. After a 2 min purging with He, the samples were heated at $300^{\circ} \mathrm{C}$ for $3 \mathrm{~min}$ and afterwards were pyrolyzed up to $600^{\circ} \mathrm{C}$, following a temperature ramp equal to $50^{\circ} \mathrm{C} / \mathrm{min}$. The $\mathrm{CO}_{2}$ trap was functioning till $390^{\circ} \mathrm{C}$. The pyrolyzed sample was further burned in an air atmosphere in the oxidation oven at $600^{\circ} \mathrm{C}$. The $\mathrm{S} 1, \mathrm{~S} 2, \mathrm{~S} 3, \mathrm{~S} 4$ values were calculated using the peak areas obtained using the PeakSimple 3.29 software, based on a previously performed calibration using standard sediment samples. The comparison of the results obtained using the above data acquisition and processing system with the ones from the RE system integrator found to be more repeatable especially when low peak areas were considered. The experimental data of RE II-TOC analysis on the original samples are shown in Table 1.

$\underline{\text { XLVII. No } 2-863}$ 


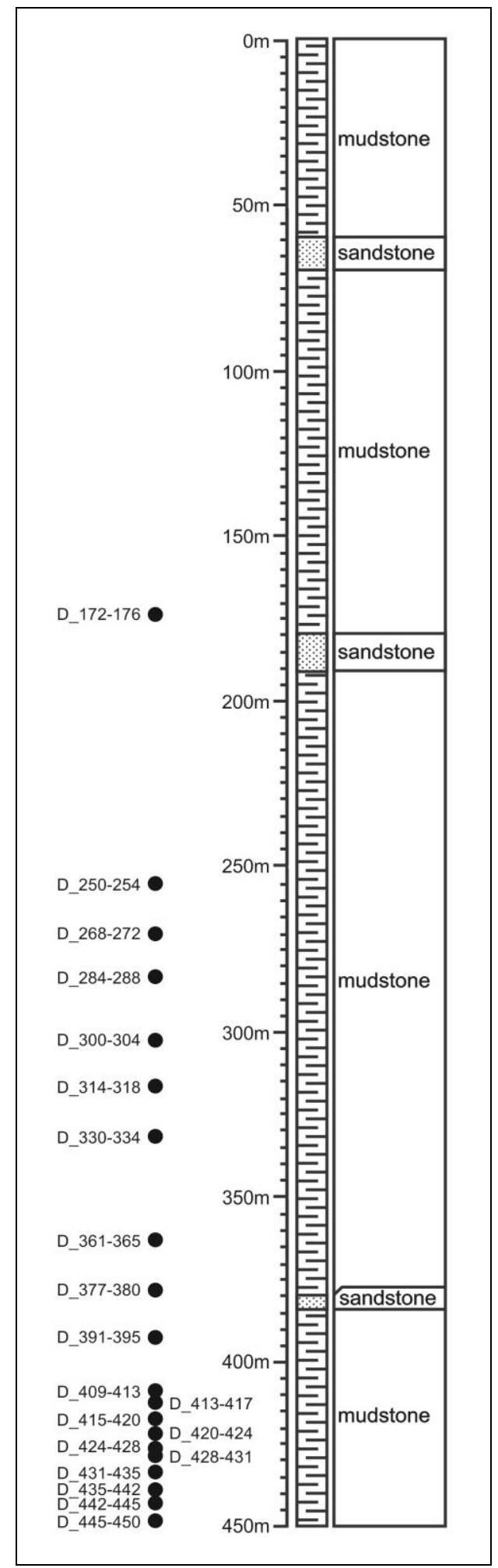

Figure 1 - Lithostratigraphic description of the well. The depths where the samples were recovered are also marked.

XLVII. No 2 - 864 


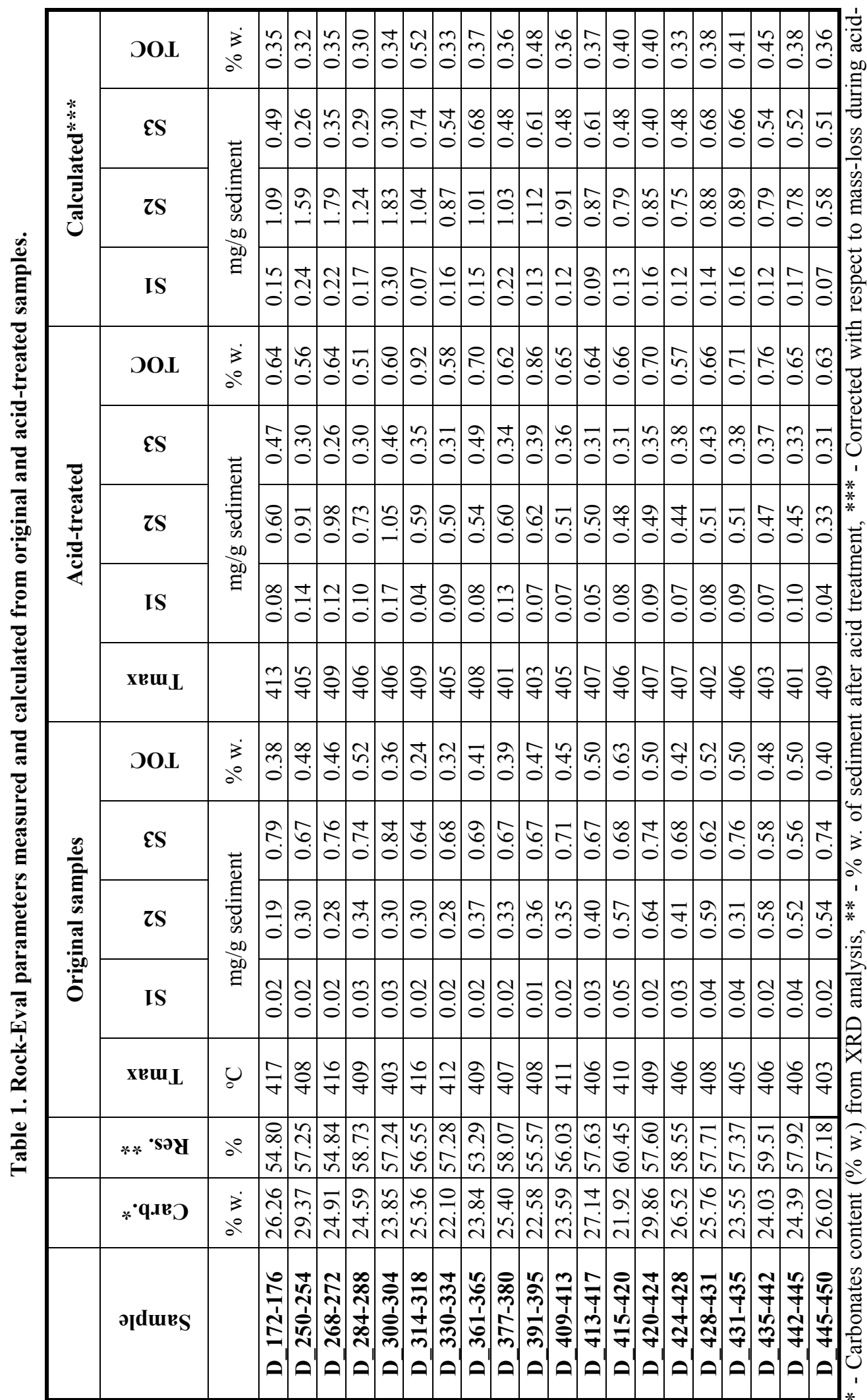

XLVII. No 2-865 
2. Subsequently the sediment samples were acid-treated to remove carbonates by applying, in a strongly repeatable way, the following methodology: Sample aliquots $(\sim 0.5 \mathrm{gr})$ were weighted and placed in centrifuge tubes. $70 \mathrm{ml}$ of $\mathrm{HCl}(2 \mathrm{~N})$ was added to each tube and they were left to react at $70^{\circ} \mathrm{C}$ in an oil bath for $12 \mathrm{~h}$. Then, after centrifuging for $5 \mathrm{~min}$ at $2000 \mathrm{rpm}$, the broth was decanted, followed by at-least five washing cycles with $50 \mathrm{ml}$ of distilled water until neutral $\mathrm{pH}$ was obtained. Specific care was taken to avoid mass-loss due to suspended particles. The remaining material was dried and weighted again to determine the sample loss due to the acid treatment. The amount of this material, expressed as fraction of the original sediment weight is shown in Table 1.

3. The two sample sets (original and acid-treated) were analyzed in a Flash 2000 Elemental Analyzer (Thermo Scientific) in CHNS mode calibrated using home-prepared standards containing carbon in low concentration (1-5\%).

4. Finally the bulk mineralogy of the samples was investigated using X-ray diffraction (XRD). XRD patterns were recorded with a Bruker D8 Advance diffractometer, with $\mathrm{Cu}-\mathrm{K} \alpha 1$ radiation, with a voltage of $40 \mathrm{kV}$ and a current of $40 \mathrm{~mA}$. The random powder mounts of samples were scanned with a step size of $0.02^{\circ} 2 \theta$ and counting time per step $0.3 \mathrm{~s}$. Raw data were evaluated using EVA software. The mineralogical composition of the samples under study is shown in Table 2 .

\section{Results and Discussion}

\subsection{RE Data Evaluation}

The examination of the RE analytical data (Table 1) shows that all studied sediment samples are immature, as their low Tmax values denote. Both original and acid-treated samples exhibited, as expected, identical Tmax values, indicating that organic content of the sediments remains unaltered during the acid-treatment, under the employed in this study experimental conditions. On the contrary, a comparison of the experimentally measured S1, S2, S3 and TOC values of both sample sets reveals significant differences, which are due to the fact that they are expressed on a different weight basis. In the acid-treated samples higher values of the organic content are obtained, because although they contain the same amount of organic matter as the original ones, their RE parameters have been calculated with respect to the remaining, after acid-treatment material, which is significantly less, as data in Table 1 show.

The removal of carbonates prior to the $\mathrm{RE}$ analysis is a common procedure in geochemical laboratories, mainly aiming to improve the accuracy of the deternination of the produced during pyrolysis of the organic matter $\mathrm{CO}_{2}$. The procedure, being not a "standard" one, is carried out under different experimental conditions in different Labs, resulting to a removal of inorganics in a different manner. Therefore the RE analytical data reported for acid-treated samples may differ significantly as they are calculated on a different weight basis. In our case, the carbonates content, determined as the sum of the calcite and dolomite from XRD analysis (Table 2), differs significantly (more than $20 \%$ w.) from the percentage of inorganics removal measured in the Lab. Therefore it can be concluded that during carbonates removal, other minerals are also removed probably dissolved in the acid solution. It is obvious that this fraction of the inorganic matter should depend heavily on the mineralogical composition of the samples under studied. Therefore it can be recommended that, RE data obtained from acid-treated samples should be corrected based on the mass-balance from the acid-treatment itself rather than using carbonates content from XRD or other suitable analytical technique. The corrected thereby data describe correctly the original samples.

\subsection{Total Carbon Mass Balance}

As reported above the original sediment samples as well as the acid-treated ones were analysed in an Elemental Analyzer and their carbon content was determined. Total carbon content (\% w.)

XLVII. No $2-866$ 
determined by EA for original and acid-treated samples, is shown in Table 3. It is obvious that the significant difference of carbon content observed between the original and the acid-treated samples is due to the presence of carbonate minerals in the inorganic matrix of the sediments. In order to check the "correctness"' of these results a mass-balance check of the measured carbon was applied. The total carbon content in the original samples may be calculated as the sum of:

1. The carbon content measured from EA on acid-treated samples (Table 3), weighted by the percentage of mass loss during treatment (Table 1).

2. The carbon content in calcite and dolomite minerals, calculated from their molecular formula and their concentration in the original samples available from XRD analysis (Table 1).

The calculated in this way total carbon content values are shown in Table 3 . These values are in good agreement with the experimentally measured from EA, with their differences exhibiting a mean relative error less than $10 \% \mathrm{w}$. This accuracy may be considered as satisfactory, keeping in mind the nature of the samples (sediments) and their possible non-homogeneity.

Table 2. Mineralogical composition of samples from XRD analysis (\% w.).

\begin{tabular}{|c|c|c|c|c|c|c|c|c|}
\hline 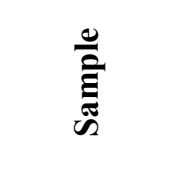 & 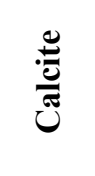 & 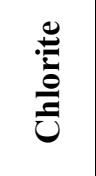 & $\begin{array}{l}\stackrel{0}{\Xi} \\
\stackrel{0}{\Xi} \\
\stackrel{0}{\circ}\end{array}$ & 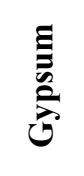 & 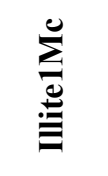 & 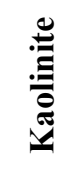 & 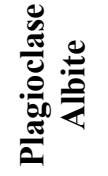 & 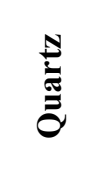 \\
\hline D_172-176 & 19.21 & 12.67 & 7.05 & 0.73 & 25.11 & 2.99 & 10.33 & 21.92 \\
\hline D_250-254 & 17.30 & 10.71 & 12.07 & 1.80 & 26.52 & 2.09 & 8.58 & 20.94 \\
\hline D_268-272 & 15.86 & 6.89 & 9.05 & 0.48 & 24.64 & 3.10 & 11.11 & 28.86 \\
\hline D_284-288 & 17.93 & 12.58 & 6.66 & 0.67 & 26.18 & 2.64 & 9.28 & 24.04 \\
\hline D_300-304 & 16.95 & 10.14 & 6.90 & 1.14 & 30.37 & 3.05 & 11.35 & 20.08 \\
\hline D_314-318 & 16.97 & 11.05 & 8.39 & 1.34 & 27.97 & 2.37 & 9.00 & 22.90 \\
\hline D_330-334 & 15.18 & 11.50 & 6.92 & 1.10 & 28.64 & 2.85 & 10.56 & 23.24 \\
\hline D_361-365 & 16.70 & 12.65 & 7.14 & 0.81 & 27.68 & 3.08 & 9.97 & 21.98 \\
\hline D_377-380 & 15.85 & 11.65 & 9.55 & 0.92 & 25.78 & 2.38 & 9.99 & 23.88 \\
\hline D_391-395 & 15.95 & 11.70 & 6.63 & 1.15 & 27.96 & 2.69 & 11.24 & 22.68 \\
\hline D_409-413 & 15.62 & 11.80 & 7.97 & 1.03 & 29.48 & 2.48 & 8.83 & 22.81 \\
\hline D_413-417 & 16.89 & 9.95 & 10.25 & 1.07 & 29.36 & 2.48 & 8.61 & 21.40 \\
\hline D_415-420 & 15.56 & 13.40 & 6.36 & 1.29 & 27.29 & 2.34 & 9.94 & 23.33 \\
\hline D_420-424 & 16.80 & 10.71 & 13.06 & 1.07 & 29.35 & 1.52 & 3.56 & 23.92 \\
\hline D_424-428 & 17.17 & 12.15 & 9.35 & 1.13 & 25.79 & 2.71 & 8.52 & 23.19 \\
\hline D_428-431 & 17.47 & 10.66 & 8.29 & 1.04 & 28.20 & 2.74 & 9.13 & 22.47 \\
\hline D_431-435 & 16.34 & 11.34 & 7.21 & 0.90 & 28.18 & 2.87 & 9.19 & 23.96 \\
\hline D_435-442 & 17.25 & 12.77 & 6.78 & 1.09 & 26.90 & 2.58 & 9.55 & 23.07 \\
\hline D_442-445 & 17.67 & 10.63 & 6.72 & 1.30 & 26.07 & 2.82 & 10.30 & 24.49 \\
\hline D_445-450 & 18.01 & 8.34 & 8.01 & 1.58 & 27.54 & 2.34 & 9.34 & 24.83 \\
\hline
\end{tabular}


Table 3. Total carbon content (\% w.) for the original and acid-treated samples by elemental analysis.

\begin{tabular}{|c|c|c|c|}
\hline 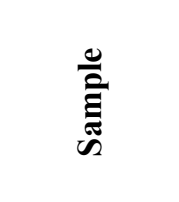 & 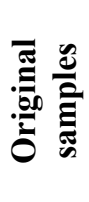 & 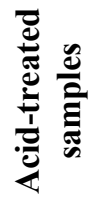 & 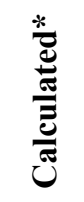 \\
\hline & \multicolumn{3}{|c|}{ Carbon content $\% \mathrm{w}$. } \\
\hline D_172-176 & 3.89 & 0.73 & 3.72 \\
\hline D_250-254 & 3.70 & 0.78 & 4.27 \\
\hline D_268-272 & 4.26 & 0.71 & 3.60 \\
\hline D_284-288 & 3.92 & 0.78 & 3.57 \\
\hline D_300-304 & 3.82 & 0.82 & 3.50 \\
\hline D_314-318 & 4.10 & 0.95 & 3.79 \\
\hline D_330-334 & 3.90 & 0.82 & 3.29 \\
\hline D_361-365 & 4.21 & 0.76 & 3.44 \\
\hline D_377-380 & 3.70 & 0.80 & 3.75 \\
\hline D_391-395 & 4.10 & 0.92 & 3.38 \\
\hline D_409-413 & 3.75 & 0.81 & 3.48 \\
\hline D_413-417 & 3.97 & 0.82 & 3.98 \\
\hline D_415-420 & 3.55 & 0.78 & 3.26 \\
\hline D_420-424 & 3.90 & 0.80 & 4.36 \\
\hline D_424-428 & 3.88 & 0.77 & 3.86 \\
\hline D_428-431 & 3.82 & 0.80 & 3.76 \\
\hline D_431-435 & 3.90 & 0.90 & 3.52 \\
\hline D_435-442 & 3.79 & 0.78 & 3.52 \\
\hline D_442-445 & 4.46 & 0.76 & 3.53 \\
\hline D_445-450 & 3.90 & 0.77 & 3.76 \\
\hline
\end{tabular}

* Corrected with respect to mass-loss during acid-treatment

\subsection{Organic Carbon Mass Balance}

Subsequently an attempt was undertaken to examine the consistence of the determined organic content values from the two employed analytical procedures (RE and EA). Initially the organic carbon content of the original sediment samples was calculated based on the measured, using EA, carbon of acid-treated samples corrected with respect to the percent of mass-loss during acidtreatment (Table 1). These values are shown in Table 4 (col. 1). Subsequently the organic carbon 
content of the samples was calculated as the sum of the measured TOC from the RE analysis of acid-treated samples, corrected for the mass-loss during acid-treatment and of the carbon content contained in the $\mathrm{CO}_{2}$ produced during pyrolysis ( $\mathrm{S} 3$ peak) of the same samples. These values are shown in Table 4 (col. 2). The pronounced agreement between the organic content values calculated from the two independent methodologies verifies their accuracy.

Table 4. Organic carbon content (\% w.) calculated from RE and EA methodologies.

\begin{tabular}{|c|c|c|}
\hline 总 & 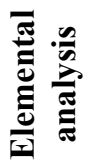 & 뜰 \\
\hline & \multicolumn{2}{|c|}{$\% \mathrm{w}$. } \\
\hline D_172-176 & 0.40 & 0.42 \\
\hline D_250-254 & 0.44 & 0.37 \\
\hline D_268-272 & 0.39 & 0.39 \\
\hline D_284-288 & 0.46 & 0.35 \\
\hline D_300-304 & 0.47 & 0.41 \\
\hline D_314-318 & 0.54 & 0.57 \\
\hline D_330-334 & 0.47 & 0.38 \\
\hline D_361-365 & 0.41 & 0.44 \\
\hline D_377-380 & 0.46 & 0.41 \\
\hline D $391-395$ & 0.51 & 0.54 \\
\hline D_409-413 & 0.46 & 0.42 \\
\hline D_413-417 & 0.47 & 0.42 \\
\hline D_415-420 & 0.47 & 0.45 \\
\hline D_420-424 & 0.46 & 0.46 \\
\hline D_424-428 & 0.45 & 0.39 \\
\hline D_428-431 & 0.46 & 0.45 \\
\hline D_431-435 & 0.52 & 0.47 \\
\hline D_435-442 & 0.47 & 0.51 \\
\hline D_442-445 & 0.44 & 0.43 \\
\hline D_445-450 & 0.44 & 0.41 \\
\hline
\end{tabular}

$\underline{\text { XLVII, No } 2-869}$ 


\section{Conclusions}

A methodology for carbonates removal from sediment samples by acid-treatment was tested and evaluated for its performance on immature sediment samples. It was verified that except of the carbonates, a significant additional fraction of the inorganic matrix was also removed during this treatment, while organic matter content of the samples was not affected.

The total carbon content of the original sediment samples, measured by EA, found to be in agreement with the one measured as the sum of the carbon content in acid-treated samples plus the carbon contained in the carbonates minerals determined quantitatively by XRD analysis.

The organic carbon content, measured using EA on acid-treated samples, is equivalent to the one determined with Rock-Eval analysis of the same samples with the addition of the carbon content in the produced $\mathrm{CO}_{2}(\mathrm{~S} 3$ peak).

\section{Acknowledgments}

This research has been co-financed by the European Union (European Social Fund - ESF) and Greek national funds through the Operational Program "Education and Lifelong Learning" of the National Strategic Reference Framework (NSRF) - Research Funding Program: THALIS -UOA"Messinian Salinity Crisis: the greatest Mediterranean environmental perturbation and its repercussions to the biota" (70/3/11605, MIS: 375405).

\section{References}

Barry J.K. 1983. Limitations of 'Rock-Eval' pyrolysis for typing organic matter, Org. Geochem. Vol. 4. No 3-4, pp. 195-199.

Behar F., Beaumont V., Penteado H.L. and De B. 2001. Rock-Eval 6 technology: performances and developments, Oil and Gas Science and Technology 56, 111-134.

Espitalie J.M., Madec B., Tissot J.J., Mennig and Leplat P. 1977. Source rock characterization method for petroleum exploration, Proceedings of the $9^{\text {th }}$ Annual Offshore Technology Conference, v.3, p. 39-448.

Lafargue E., Marquis F. and Pillot D. (1998). Rock-Eval 6 Applications in Hydrocarbon Exploration, Production and Soils Contamination Studies, Oil \& Gas Science and Technology, 53, 4, 421-437. 